$16^{\text {th }}$ International Conference on

AEROSPACE SCIENCES \& AVIATION TECHNOLOGY,

$\boldsymbol{A S A T}$ - 16 - May 26 - 28, 2015, E-Mail: asat@mtc.edu.eg

Military Technical College, Kobry Elkobbah, Cairo, Egypt

Tel : +(202) 24025292 - 24036138, Fax: +(202) 22621908

\title{
Development of high Precision Multi-Spectrum Tracking System
}

\author{
Ashraf Aboshosha, \\ Radiation Eng. Dept., NCRRT, Atomic Energy Authority, Nasr City, Cairo Egypt \\ ashraf.shosha@gmail.com
}

\begin{abstract}
:
Throughout this framework the pattern analysis and recognition has been accomplished based on the fusion of spatial and spectral sensorial data. The main purpose of that is the exploration of some salient features in the spatial and spectral domains that simplifies the pattern recognition. The spatial and spectral fusion of data patterns confirms the matching process that results where Bayesian rule has been applied to the available heterogeneous data. To prove the proposed technique, several visible and IR images have been used to show the possibility of integrating several spectral range images to achieve the pattern recognition. Also, this approach has been applied to support a too critical forensic purpose, it is the premature burial.

Keywords: Spatial domain, Spectral domain, Data fusion, Visual image processing, IR image processing, forensic image processing, premature burial
\end{abstract}

\section{INTRODUCTION}

Extraction of salient features in data patterns is a very important issue for recognition, classification and similarity measure applications where the pattern size and data amount could be huge. The saliency here means the uniqueness of a certain feature by which the comparison between the patterns could be clear and expressive.

Fusion of spatial and spectral sensorial data is an essential subject in high tech applications such as; concealed weapon detection, night time surveillance, automatic landing system, digital camera applications, medical diagnosis, defect inspection and remote sensing

The goal of spectral and spatial fusion is to integrate multi-sensor, multi-temporal and/or multi-view data into one new pattern containing information, the quality of which cannot be accomplished otherwise. This research perform multi-sensor fusion of visual and infrared (IR) patterns. Hence, in night-vision environment, only limited visual data can be captured by cameras in poor lightning. In such conditions it is difficult to do surveillance only by visual sensor while IR camera, that is IR sensor, captures thermal pattern of object clearly. Thermal pictures of pedestrian in night-vision environment can be seen clearly in IR pattern sequence. IR patterns provide rich data for hot objects, but poor data for cold ones. Visual spectrum, on the other hand, provides the visual context to the objects. Thus, the fusion of the two spectra spatially will provide good perceptibility to human vision under poor lightning condition. This will help detect the moving objects whatever the circumstances perfectly. Several research works have been presented in this area of study. Anjali Malviya, S. G. Bhirud presented a fusion technique for visible and IR images. J. Heo, S. Kong, B. Abidi, and M. Abidi discusses the fusion of visual and thermal signatures with a focus on eyeglass removal for the purposes of face recognition $[1,2,3,4]$. 
Pattern recognition using visual images is gaining attention as a superior biometric. Images taken from visual band are formed due to reflectance. So, they depend on the external light source, which sometimes might be absent e.g. night vision or when there are heavy clouds. Imagery is also difficult because it depends on the intensity of light and the angle of the incident of light. Commonly used face recognition techniques are discussed in [5-10]. Recently, face recognition on thermal infrared spectrum has become more common because thermal pattern is formed due to thermal emission not light reflection. They do not depend on the external light source and the intensity of the light and are also less dependent on the angle of the incident of the light. Different methodologies of pattern recognition are performed on visual/thermal images and are compared in [5-10]. Pattern fusion can be divided into three types namely, pixel level, feather level and decision level. Both feather level and decision level pattern fusion may result in inaccurate and incomplete transfer of information [5-10].

Pattern recognition is a challenging task and its domain of application is extended, covering different areas such as security systems, defense applications, and intelligent machines. It involves different image processing issues such as face detection, recognition and feature extraction. Recently, fusion of pattern images became more common as a superior biometric in case of face recognition. Data fusion is a method which combines different patterns of data gathered by the simultaneous use of several sensors to generate a new type of data. Different perceptual mechanisms integrate these senses to produce the internal representation of the sensed environment. The fusion tends to be synergistic in the scene that data inferred from the process cannot be obtained from any proper subset of the sense modalities. This feature of synergism is one that should be sought for when applying multisensory integration for machine perception. The principal motivation for the fusion approach is to exploit such synergism in the technique for combined interpretation of images obtained from multiple sensors. Hence, research work on fusion has been carried out for years. And the obtained fusion results can be classified into two types. One is about weak fusion methods, and the other is about strong fusion methods. In the first Type of fusion methods, fusion of data generated by sensory modules does not affect the operation of the modules. On the contrary, for strong fusion methods, the modules producing the information to be fused are being affected in some way by other information from other modules. For the detailed review on current advances in visual and thermal pattern recognition. Simple image fusion in spatial domain is discussed earlier, where pattern recognition is used to test the fusion of pattern database patterns. Both pattern fusion and decision fusion is employed to improve the accuracy of the pattern recognition system [11-18].

The paper is organized as follows: Fast Spectral Analysis of visible and IR ranges is presented in section II. The Spectral Fusion for Forensic Death Diagnosis is discussed in section 3. Section 4 presents the conclusions.

\section{Spectral Analysis of visible and IR ranges}

Infrared light lies between the visible range and microwave ranges of the electromagnetic spectrum. It has a range of wavelengths, just like visible light has wavelengths that range from red light to violet. "Near infrared" light is the closest light in wavelength to visible light and "far infrared" is closer to the microwave range of the electromagnetic spectrum. The pictures in figure (1.a and 1.b) show the slight difference between the near IR to visible range. Figure 2 shows face recognition with and without glasses based on multi-spectrum integration.

Far infrared waves are thermal. In other words, humans experience this type of infrared radiation every day in the form of temperature. The heat that we feel from the sun, a fire, a radiator or a warm things in general is infrared. The temperature-sensitive nerves in our skin can detect the difference between inside body temperature and outside temperature. Shorter, near infrared waves are not hot. In fact we cannot even feel them. These shorter wavelengths are the ones used by TV's remote control [19-22].

\subsection{Direct Fusion of IR and visual Spectra}

This research work fused visual and thermal images. Ideally, the fusion of common pixels can be done by pixelwise weighted summation of visual and thermal images.

$$
F(x, y)=a(x, y) V(x, y)+b(x, y) T(x, y)[12] \ldots
$$

where $F(x, y)$ is a fused output of a visual image, $V(x, y)$, and a thermal image, $T(x, y)$,

while $\mathrm{a}(\mathrm{x}, \mathrm{y})$ and $\mathrm{b}(\mathrm{x}, \mathrm{y})$ represent the weighting factors for visual and thermal images respectively. This work considers $\mathrm{a}(\mathrm{x}, \mathrm{y})=0.70$ and $\mathrm{b}(\mathrm{x}, \mathrm{y})=0.30$

To show the difference between single spectrum and image fusion based on different of segmentation templates. Table (1) presents two case of study of face recognition with and without glasses while figure 2 shows the face detection based on spectral and spatial fusion of IR and visible images 


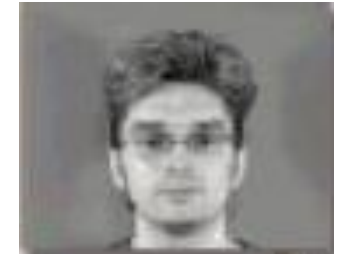

(a) Visible range imagery

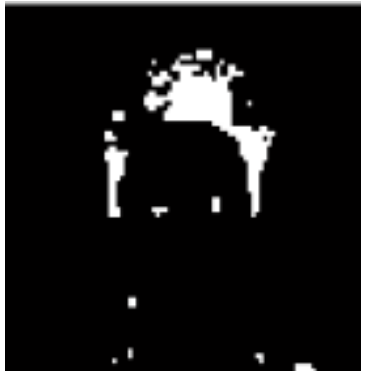

(c) Mismatch of visible spectral pattern match due to similarity/affinity

Figure 1: The integration among different spectral ranges reinforces the precision of the pattern recognition

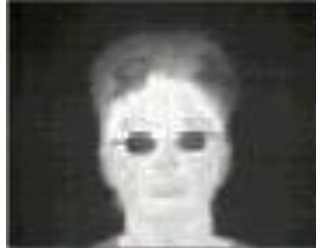

(b) IR range imagery

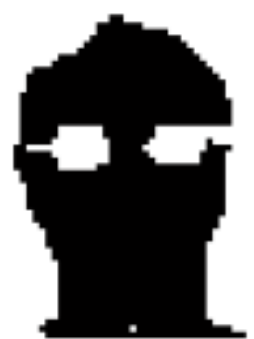

(d) Uniqueness of IR spectral pattern matching without glasses

Table 1, Case1:

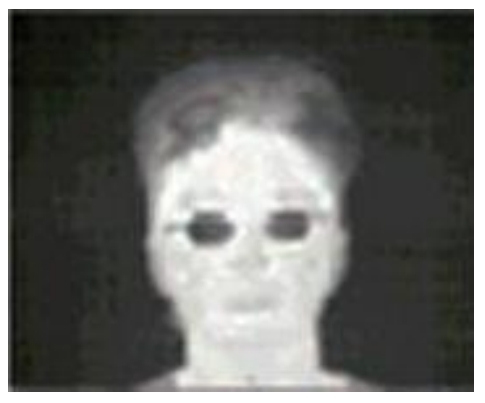

Original IR Image

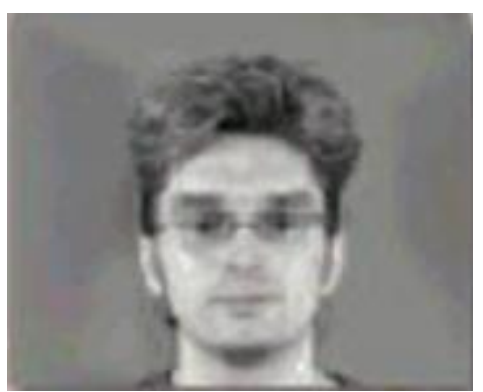

Original visible Image

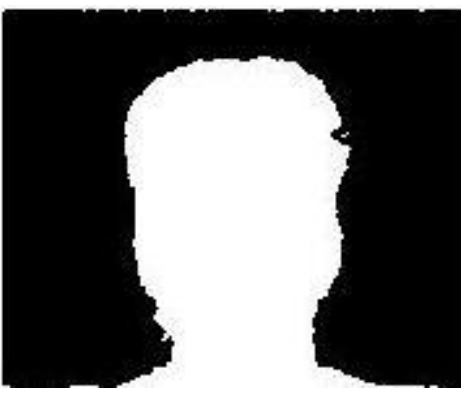

Binary segmented Image based on IR templete

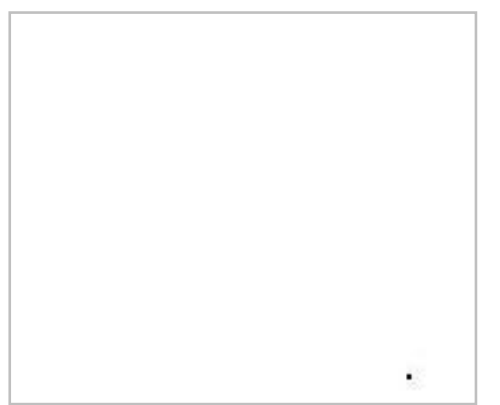

Binary segmented Image based on visual templete

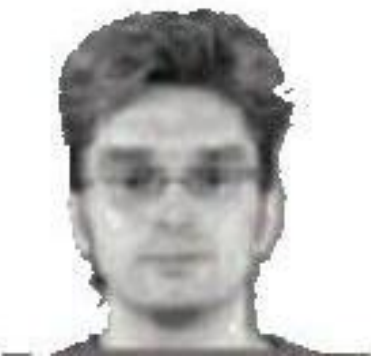

Successful face detection based on the fusion of IR and Visual Images. Exact detection with glasses.

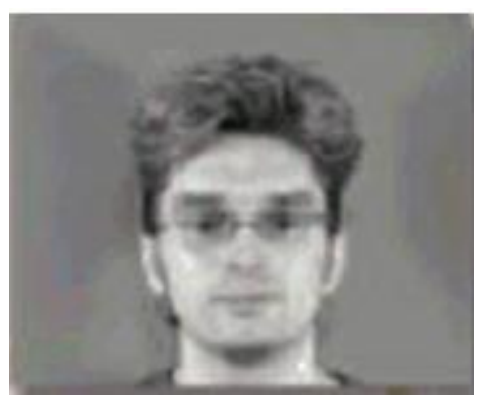

Failed face detection based on the visual segmentation.

Successful face detection based on the fusion of IR and Visual Images. Exact detection with glasses 

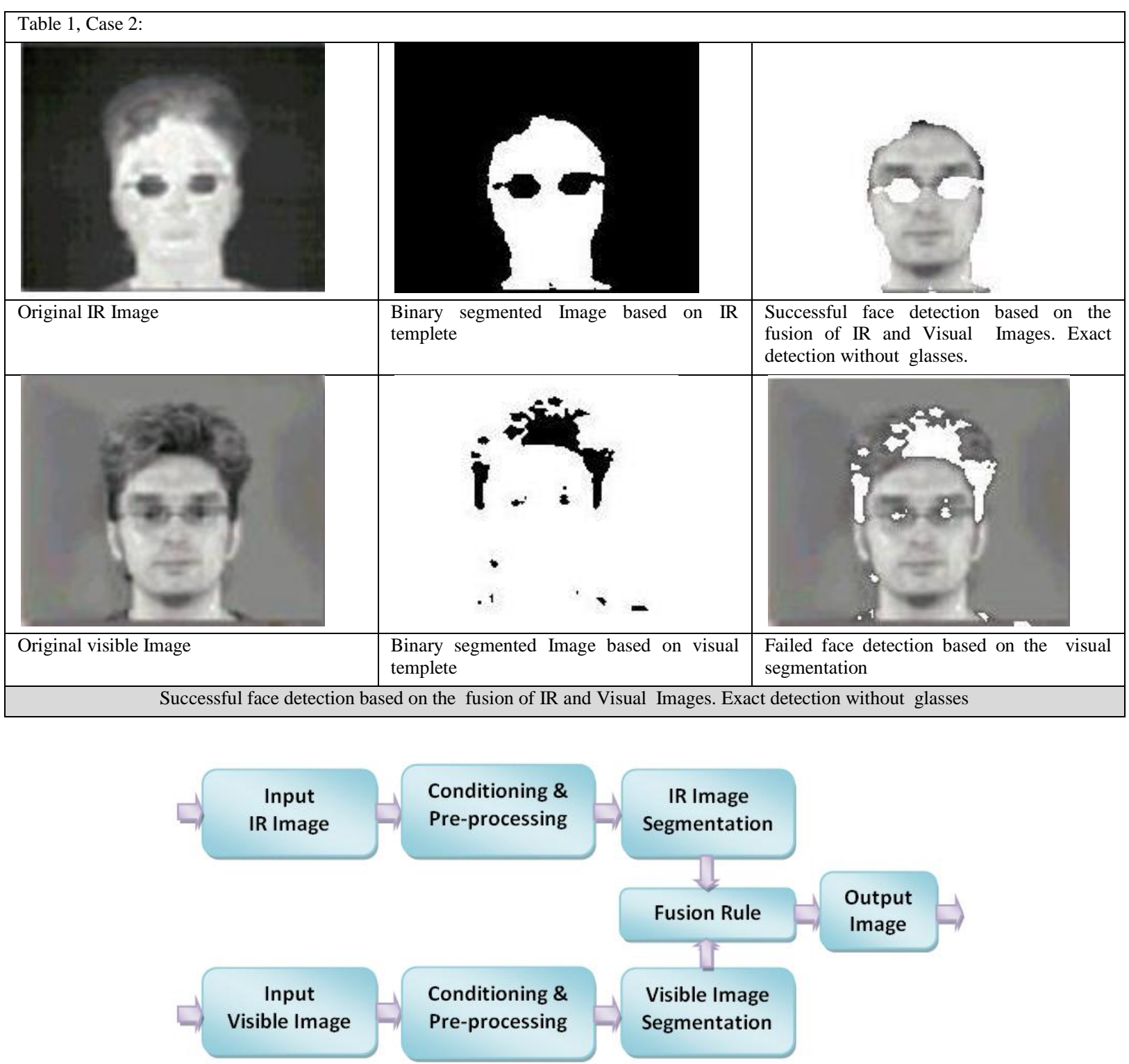

Figure 2.: Face detection based on spectral and spatial fusion of IR and visible images

The fusion rule used here is based on feature level. The data obtained from one spectrum is used to extract the feature vector from the other spectrum which are independent from those extracted from the other, these feature vectors are concatenated to produce a single new fused pattern. This process is difficult when spectra are heterogeneous. One of the most important factors is the selection of the appropriate IR rage for precise face detection. Figure 3 shows the results of face detection based on different IR ranges. The results confirm that the fusion of visible images with the Mid-Wave IR gives the best face detection. 


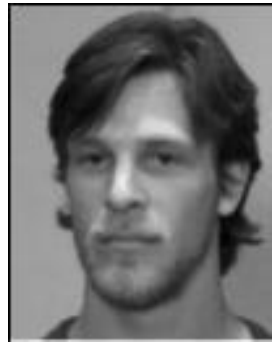

Visible Range

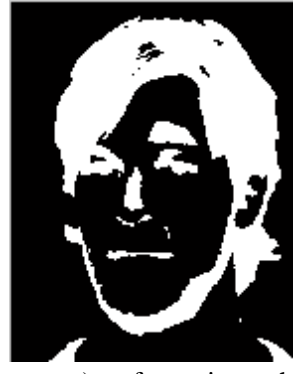

a) face mismatch

0.5 : 0.7 Microns

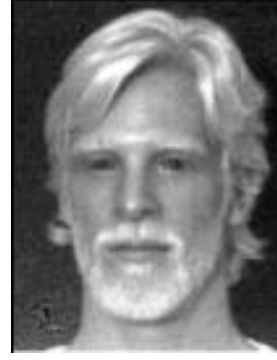

Short-Wave (near) IR

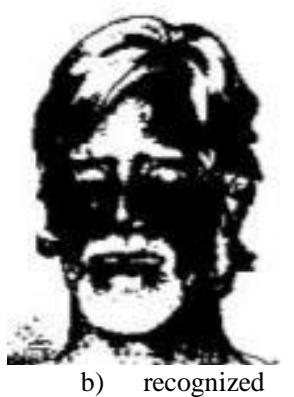

$1.0: 2.0$ Microns

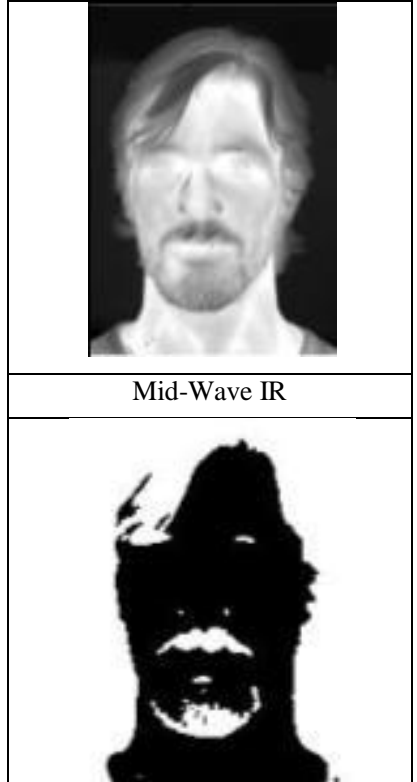

c) recognized

$3.0: 5.0$ Microns

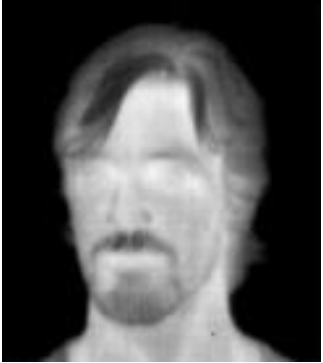

Long-Wave (far) IR

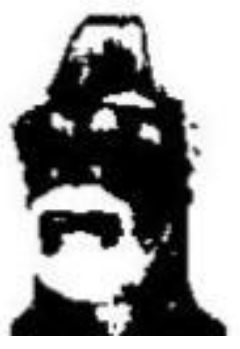

d) recognized

$8.0: 12.0$ Microns

Figure 3.: Pattern fusion analysis over visible and IR ranges. The best result is obtained from the integration of visual and mid-wave IR

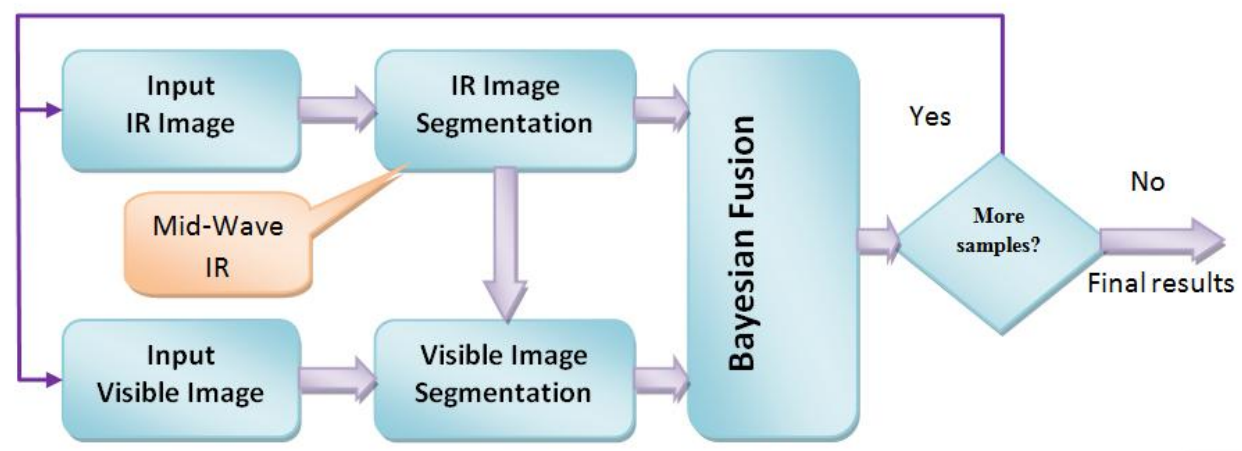

Figure 4.: Face recognition based on spectral and spatial fusion of IR and visible images using Bayesian Fusion

\subsection{Bayesian Fusion}

The Bayesian Update form makes the probability of an object being a face (Pl) is one minus the probability of it being false $(\mathrm{Pd})$, and vice-versa, so;

$P l(x, y)=1-\operatorname{Emp}(x, y)=\operatorname{Map}(x, y)$

Figures 4. Presents face recognition based on spectral and spatial fusion of IR and visible images using Bayesian fusion.

If the pattern is in the free space area of the camera (either visual or IR), the value $P_{E}(x, y)$ is generated. The first step is to translate $P_{E}(x, y)$ so that rather than representing the probability that a pattern is a face of person using number is the range [0..1], the range of numbers [0..0.5] is used, where zero means that the pattern is definitely not a face of person, and 0.5 means there is no information about the object.

$P_{E}(x, y)^{\prime}=\left(1-P_{E}(x, y)\right) / 2$

$P_{E}(x, y)$ is then integrated with the prior value of the pattern using the equation below, the Bayesian probabilistic update formula.

$\operatorname{Map}(x, y)=\frac{P_{E}(x, y) * \operatorname{Map}(x, y)}{P_{E}(x, y) * \operatorname{Map}(x, y)+\left(1-P_{E}(x, y)\right) *(1-\operatorname{Map}(x, y))}$

A similar method is performed on an update on a pattern in the area of the camera. First $P_{o}(x, y)$ is translated from the range [0..1] to [0.5..1], where 0.5 represents deficit of knowledge about the object, and 1 means the object is definitely correct. 
$P_{o}(x, y)^{\prime}=0.5+P_{o}(x, y) / 2$

Finally the map is updated by the new value:

$\operatorname{Map}(x, y)=\frac{P_{o}(x, y) * \operatorname{Map}(x, y)}{P_{o}(x, y) * \operatorname{Map}(x, y)+\left(1-P_{o}(x, y)\right) *(1-\operatorname{Map}(x, y))}$

The Log Update Method uses a multiplication to integrate new values from the cameras with a priori data, and then combines the probabilities of the object being object or not using logarithms and addition. Because of the use of multiplication, it is required for $P_{O}(x, y)$ to be translated to a number greater than one, and for $P_{E}(x, y)$ to be translated in a similar manner to a number less than one. This ensures that a surface reading causes the overall value to rise, while a free space reading causes it to fall.

$$
\begin{aligned}
& P d(x, y)^{\prime}=P d(x, y) * P_{E}(x, y) \\
& P l(x, y)^{\prime}=P l(x, y) * P_{O}(x, y)
\end{aligned}
$$

Finally, the free space and surface values are combined to form the result using logarithms and addition.

$$
\operatorname{Map}(x, y)=\log _{10}(P d(x, y)) * \log _{10}(P l(x, y))
$$

Unlike the previous two mathematical update methods, the Log Update Method does not map values into a range [0..1]. For this reason a cut off value must be set at which it can be said that an object is definitely alive and another value at which the object is definitely dead.

\section{Spectral Fusion for Forensic Death Diagnosis}

One of the most important applications of the spatial and spectral analysis of signals is the victim detection in disasters. When one looks at a picture of the human body with infrared technology, all you see is radiating heat. That heat is generated within our bodies and it helps to keep us alive. In fact, the human body generates so much heat that in only 30 minutes, the average body gives off enough heat (throughout the entire body) to bring half a gallon of water to boil. To detect alive bodies the thermographic imagery is an efficient technique to distinguish between dead and alive bodies. Figure (5) shows the vanishing of thermal energy radiated by a body from alive state to death state.

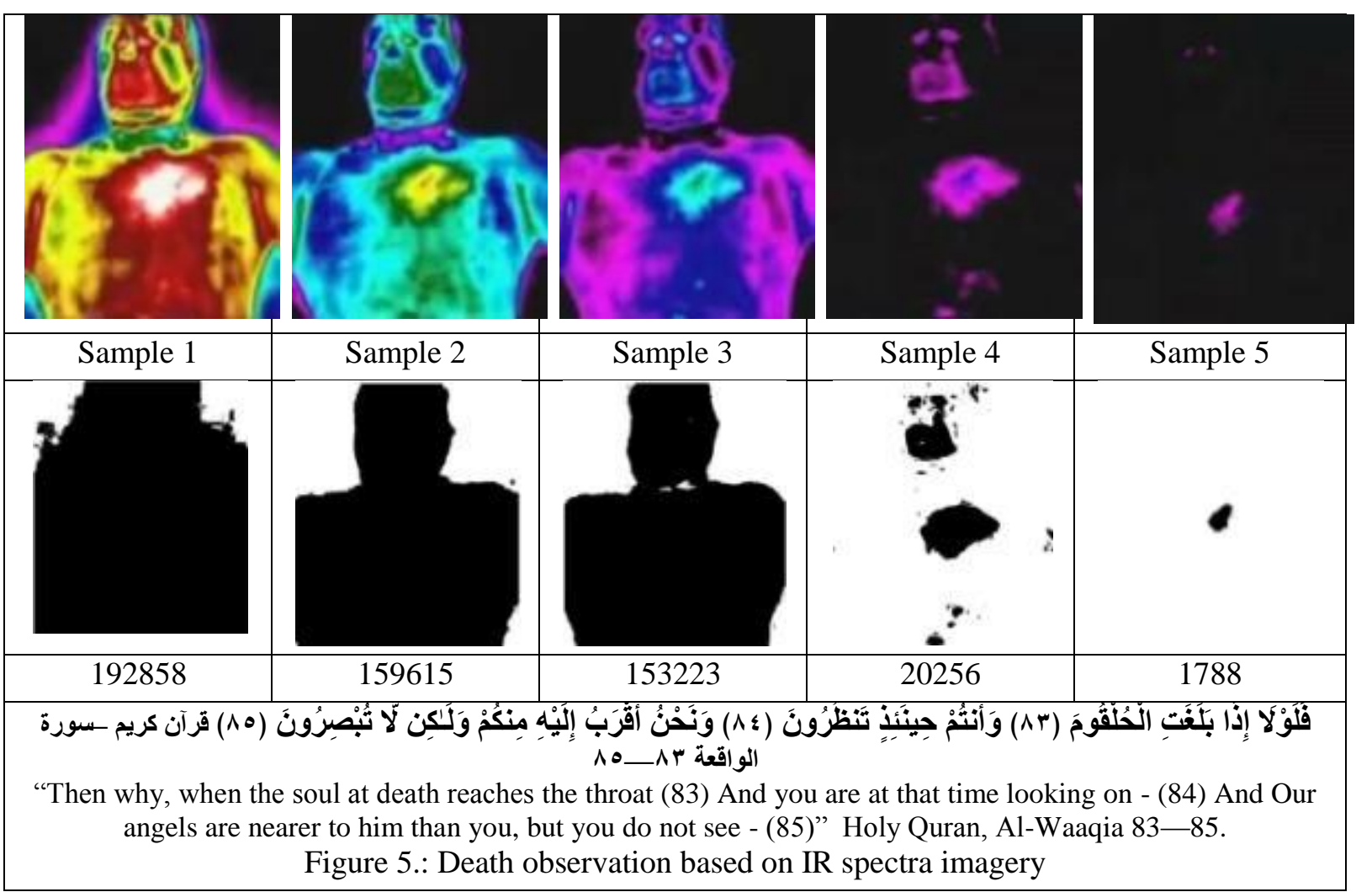

Death process has emotional, spiritual, mental and physical symptoms. Throughout this article we focus on employing spatial and spectral image analysis to study the death process by which the right treatment could be 
applied. The visual symptoms are clear signs of death where the skin color changes and coldness of the body increases over the time. Figure shows the gradual cooling of dead body under IR imagery. The visual diagnosis may provide a clear decision to distinguish among death symptoms and the symptoms of some toxicity conditions where the patient appears to be dead while he/she is still alive. This framework supports forensic purposes by helping in avoiding misdiagnosis of death and preventing premature burial. Figure 5. shows the gradual cooling of a copse based on the infrared imagery.

\section{CONCLUSIONS}

Throughout this framework the pattern analysis and recognition has been accomplished based on the fusion of spatial and spectral sensorial data. The presented results show the improvement and precision in face recognition based on the integration of both visual and IR images. To distinguish between the decision making and decision confirmation Bayesian rule has been applied to confirm the results based on repeated visual and infrared images. Moreover the presented approach has been applied to support a very critical subject in forensic research, it is premature burial, where we recommend that in some conditions the corpse of someone should not be buried or cooled in refrigerators before 24 hours of the physical death declared by a physician.

\section{References}

[1] J. Heo, S. G. Kong, B. R. Abidi and M. A. Abidi. "Fusion of Visual and Thermal Signatures with Eyeglass Removal for Robust Face Recognition". Conference on Computer Vision and Pattern Recognition Workshop (CVPRW'04) Vol. 8. pp. 122-122. USA. 2004.

[2] J. Heo, S. Kong, B. Abidi, and M. Abidi, "Fusion of Visual and Thermal Signatures with Eyeglass Removal for Robust Face Recognition" IEEE Workshop on Object Tracking and Classification Beyond the Visible Spectrum in conjunction with CVPR 2004, pp. 94-99, Washington, D.C., July 2004.

[3] Jingu Heo, "Fusion of Visual and Thermal Face Recognition Techniques: A Comparative Study", The University of Tennessee, Knoxville, October 2003, http://imaging.utk.edu/publications/papers/dissertation.

[4] Anjali Malviya, S. G. Bhirud, "Visual Infrared Video Fusion for Night Vision using Background Estimation”, JOURNAL OF COMPUTING, VOLUME 2, ISSUE 4, pages (66--69) APRIL 2010, ISSN 2151-9617.

[5] I. Pavlidis, P. Symosek, B. Fritz. "A Near Infrared Fusion Scheme for Automatic Detection of Humans". U.S. Patent Pending. H16-25929 US. filed in September 3, 1999

[6] A. Gyaourova, G. Bebis and I. Pavlidis. "Fusion of infrared and visible images for face recognition". Lecture Notes in Computer Science. 2004.Springer. Berlin.

[7] A. Selinger, D. Socolinsky. "Appearance-based facial recognition using visible and thermal imagery: a comparative study". Technical Report 02-01. Equinox Corporation. 2002

[8] M. Hanif and U. Ali. "Optimized Visual and Thermal Image Fusion for Efficient Face Recognition". IEEE Conference on Information Fusion. 2006

[9] G. Bebis, A. Gyaourova, S. Singh and I. Pavlidis. "Face Recognition by Fusing Thermallnfrared and Visible Imagery". Image and Vision Computing. Vol. 24, Issue 7. July 2006. pp.727-742

[10] A. Ross and A. Jain. "Information fusion in biometrics". Pattern Recognition Letters. Vol. 24 , No. 13. pp.2115-2125. 2003

[11] A. Singh, G. B. Gyaourva and I. Pavlidis. "Infrared and Visible Image Fusion for Face Recognition". Proc. SPIE. vol.5404. pp.585-596. Aug.2004

[12] Hanif, M.; Ali, U., “Optimized isual and Thermal Image Fusion for Efficient Face Recognition”, 2006 9th International

[13] Conference on Information Fusion, proceedings of IEEE, Volume, Issue, 10-13 July 2006 Page(s): 1-6

[14] [9] Chen, Y. Blum, R.S., "Experimental Tests of Image Fusion for Night Vision", 2005 8th International Conference on

[15] Information Fusion, proceedings of IEEE, Volume: 1, On page(s): 8 pp.

[16] David Hughes, "Sinking in a Sea of Pixels- The Case for Pixel Fusion", 2006 Silicon Graphics, Inc.,

[17] http://www.sgi.com/pdfs.

[18] Zhang Yin, Andrew A. Malcolm, "Thermal and Visual Image Processing and Fusion", http://www.simtech.astar.edu.sg/Research/TechnicalReports.

[19] Taj, J. A., Ali, U., Qureshi, R. J., Khan, S.A., "Fusion of Visual and Thermal Images for Efficient Face Recognition using Gabor Filter", accepted for publication in Proc. Of The 4th ACS/IEEE International Conference on Computer Systems and Applications, March 8-11, 2006, Dubai/Sharjah, UAE. 
[20] Ali, U., Taj, J. A. "Gabor Filter based Efficient Thermal and Visual Face Recognition Fusion Architecture" accepted for publication in the fourth International Conference on Active Media Technology, June 7-9 2006 Brisbane, Australia.

[21] E. J. Bender, C. E. Reese, and G. S. van der Wal, "Comparison of additive image fusion versus featurelevel image fusion techniques for enhanced night driving”, Proceedings of SPIE, Vacuum and Solid State Photoelectronic Imagers, Detectors, and Systems, 4796, 2002.

Z. Zhang and R.S. Blum, "A categorization of multiscale-decomposition-based image fusion schemes with a performance study for a digital camera application”, Proceedings of IEEE, 87(8):1315-1326, 1999. 